\title{
Tutorial on Design Verification with Synchronized Transitions
}

\author{
Niels Mellergaard and Jørgen Staunstrup \\ Department of Computer Science, Technical University of Denmark, \\ DK-2800 Lyngby, Denmark \\ email: $\{\mathrm{nm}, \mathrm{jst}\} @$ id.dtu.dk
}

\begin{abstract}
This tutorial describes a mechanized technique for design verification. The aim is, in the early design phases, to verify selected key properties of a partially specified design. A supporting design language called SYNChronized Transitions is used for describing designs. The design verification is mechanized by tools, in particular, a theorem prover called the Larch Prover (LP) used for reasoning about properties of a design, and a translator (called ST2LP) that generates input for the theorem prover from a given design description.
\end{abstract}

\section{Introduction}

Design verification aims at the initial phases of a circuit design where descriptions of the design are incomplete and abstract. This tutorial introduces a design verification technique and supporting tools for verifying selected key properties of a partially specified design, e.g., that the design meets a safety requirement, or that its communication with the environment meets a certain protocol. Recently, there has been widespread interest in formalized hardware verification, see [9] for a survey. A major part of this work is oriented towards implementation verification $[3,8]$ where a high-level description (the specification) is related to another lower level description (the realization). Implementation verification is necessarily aimed at a later stage of design where both a specification and an implementation are available. In contrast, design verification, aims at verifying particular properties of an incomplete design; possibly long before any implementation is started.

To encourage early verification, it is important to enable the designer to carry out the verification on exactly the same design description that is later used for other purposes, e.g., synthesis or simulation. One way to achieve this is to develop tools and transformation techniques that allow such manipulations on design descriptions formulated in a logic intended for verification $[6,11]$. The approach described in this tutorial is somewhat different; by supporting verification in a design language that is not specifically aimed at verification. Hence, a design description can be used for a number of purposes, e.g., simulation, synthesis, performance analysis etc.

The design verification technique is supported by mechanical tools: a translator and a theorem prover. The translator transforms design descriptions specified 
with SYNCHRONIZED TRANSITIONS [14], including formally stated requirements to the design, into verification conditions for a mechanical theorem prover (the LARCH Prover, LP [10]). The translation is syntax directed which means that the structure of the verification follows the structure of the design description. The verification is broken into a large number of small steps, each syntactical construct yields a constant number of verification conditions (for some zero).

A high-level description of a design is an abstraction of its physical behavior. It is important to realize that formal verification deals with the abstraction and not with the physical realization. A formal verification of a requirement is not an absolute guarantee against malfunctioning. For example, in case of a power failure the formal description is no longer a model of the physical realization, and hence, properties verified from the description may no longer hold. The same applies to simulation which is also based on a model of the physical reality. If the model is not an adequate abstraction, a simulation, no matter how exhaustive, does not provide any guarantee against malfunctioning. It is outside the realm of formal methods to ensure that a model adequately reflects physical phenomena such as light or changing voltages. Despite these reservations, formal verification can be a very powerful tool, but it is important to realize that it can never give complete assurance.

\section{Synchronized Transitions}

A design in SYNChronized TRANSiTions describes a computation as a set of independent, concurrently executing transitions. These transitions communicate via (shared) state variables. In addition to these two fundamental concepts: transitions and state variables, SYNCHRONIZED TRANSITIONS has language constructs for expressing initialization, parameterization, protocols, invariants, hierarchy (cells), etc. In Sect. 5, the language is presented through an example. There are strong conceptual similarities between SYNCHRonized Transitions and UNITY, as developed by Chandy and Misra [4]. Both describe a computation as a collection of atomic conditional assignments without any explicit flow of control. Chandy and Misra propose this as a general programming paradigm. Our application of SYNCHRONIZED TRANSITIONS is more specialized, in particular, the development of application specific integrated circuits. The main difference is the structuring concepts of SYNCHRONIZED TRANSITIONS: cells, parameters, statics, etc., in addition, there is a number of syntactical differences.

\section{Mechanical Theorem Proving}

There are many CAD tools available to help designers master the complexity of large designs. Most of these tools are aimed at a low level, e.g., at the layout or netlist level. There is a similar need for tools to assist in doing high-level design. This tutorial describes such tools that are used for verification.

Currently, research is going on with different approaches for verification, e.g., model checking and theorem proving (see [9] for a survey). The tools described in 
this tutorial are based on theorem proving, where a proof consists of a number of verification steps. To verify a large design, many verification steps are required. It becomes quite tedious to do this by hand, and practical experience with the approach described here tells that the verification rarely requires much insight or mathematical sophistication. Furthermore, the verification must be redone every time a design description is changed. By using a mechanical theorem prover, it is possible to reduce the routine work needed to do formal verification. There is a number of mechanical theorem provers available. They differ significantly, for example, in the expressive power of the notation allowed for stating a conjecture. However, the notion of proof used in all the currently available mechanical theorem provers is rather similar. A proof is a series of reductions transforming the conjecture into a formula known to be true (or false). The reductions are either built into the theorem prover or supplied by the user. For example, once a conjecture has been verified, it can be used to reduce other conjectures. This mechanical notion of a proof as something consisting of a finite number of applications of reductions is somewhat more restricted than the notion of proof usually found in mathematics. However, verification of most interesting properties of real designs does not require deep mathematical insight and, therefore, the mechanical notion of proof is usually adequate. When this is said, it is also acknowledged that for nontrivial designs, a significant effort is needed to do a formal verification. This is not only a question of finding a successful proof. Often the outcome is the detection of bugs in the design description; either in the explicitly stated requirements of the design, or in the algorithm implemented by the transitions.

The theorem prover LP [7] has been used in experiments with mechanized verification of designs in SYNCHRONIzED TRANSITIONS. LP is a theorem prover for a subset of multi-sorted first-order logic. It is designed to work efficiently on large problems and to be used by relatively naive users. LP is intended primarily for use as an interactive proof assistant, not as a fully automatic theorem prover. Its design is based on the assumption that initial attempts to state conjectures correctly, and then to prove them, usually fail. As a result, LP is designed to carry out routine (and possibly lengthy) steps in a proof automatically and to provide useful information about why proofs fail, if and when they do. Unlike the Boyer-Moore prover $[1,2]$, it does not use heuristics to formulate additional conjectures in a search for a proof that may not exist. Strategic decisions, such as trying induction, must appear as explicit LP commands (either entered by the user or generated by an application-specific front-end). When proof attempts fail, LP provides quick feedback; in fact, if and when time-consuming proof attempts occur, they are generally a sign that something is wrong.

The choice of a first-order logic has not been significant for the overall approach, but merely directed by the theorem prover. LP is used mainly of historical reasons; the axiomatization of conjectures and facts might be more elegant in a higher-order logic, however, the emphasis has been on providing efficient machine assistance, and LP has this far satisfied the needs. 


\section{Automatic Translation}

A translator, called ST2LP, is used for translating design descriptions into verification conditions in the format required by LP. It extracts appropriate declarations for state variables, axioms defining transitions, protocols, invariants, and proof obligations from design descriptions. When formalizing conjectures, the translator often generates fruitful idioms for the proof. For example, when formalizing invariance proofs, the translator generates an LP command to initiate a proof by cases on the transition selected for execution. As a result of these idioms, LP often verifies invariants of designs directly from the output of the translator without any manual assistance. In many cases, when proofs are not completely automatic, there is a number of commands and standard techniques that can push proofs through manually. In the tutorial [16] it is shown how LP is used to do mechanical proofs based on proof scripts generated by ST2LP.

\section{Black Jack Dealer}

The Black Jack dealer is one of the benchmark examples in the collection distributed for the TPCD ${ }^{1}$ conference. This example is used to introduce the notation and key concepts of SYNCHRONIZED TRANSITIONS.

A Black Jack dealer at a casino plays his own hand according to a completely predetermined procedure that leaves no possibility for human choice. This example presents a mechanical Black Jack dealer which plays the dealer's hand. Its inputs are cardready (true/false) and card (2 of Clubs, ..., Ace of Spades). Its outputs are hitme, stand, and broke (all truth-valued). The cardready/hitme signals are used for a four-phase handshake with the operator. Cards are valued from 2 to 10 (court cards have the value 10), and aces may be valued as either 1 or 11. The Black Jack dealer is repeatedly presented with cards. It must assert stand when its accumulated score reaches 17 ; and it must assert broke when its score exceeds 21 . In either case the next card starts a new game.

\subsection{Transitions and State Variables}

A design is modeled in Synchronized Transitions as a collection of transitions operating on a set of state variables, for example:

$$
\ll \text { idle } \wedge \neg(\text { broke } \vee \text { stand }) \wedge \text { cardready } \rightarrow \text { idle: }=\text { FALSE } \gg
$$

This transition operates on the state variables idle, broke, stand, and cardready; it allows $i d l e$ to change (to false) when the precondition $i d l e \wedge \neg($ broke $\vee$ stand $) \wedge$ cardready holds. All four state variables are boolean. Another transition is:

$$
\ll \neg \text { idle } \wedge \neg \text { cardready } \rightarrow \text { idle: }=\text { TRUE } \gg
$$

This allows idle to become true when the precondition holds. Operationally, the computation can be modelled as repeated nondeterministic selection and execution of an enabled transition. In this model, transitions are executed:

\footnotetext{
1 Theorem Provers in Circuit Design
} 
- concurrently,

- repeatedly, being ready for another execution immediately upon completion,

- atomically, as indivisible operations, and

- independently of the order they appear in the design description.

It is not required that a transition is executed immediately after it becomes enabled, because other enabled transitions may be selected. In fact, there is no upper bound on when a transition is selected. This models delays caused by the components in physical circuits. SYNCHRONIZED TRANSITIONS is not intended for specifying and dealing with explicit timing, for example, verifying that a given timing constraint is met. The verification results hold independently of the actual delay.

This is a very concrete and simple model of a computation, and it must be stressed that it is only a model, and a realization may execute transitions differently, as long as the realization does not enter states that cannot be entered by the model. As explained in [14], it is often possible to ensure atomicity, even if several transitions are executed in parallel, thus allowing efficient circuit realizations where many sub-circuits operate simultaneously.

\subsection{Initial State}

The initial state of a computation, i.e., the initial value of state variables is specified as follows:

$$
\begin{aligned}
& \text { INITIALLY } \\
& \text { idle }=\text { TRUE } \\
& \text { hitme }=\text { FALSE } \\
& \text { stand }=\text { FALSE } \\
& \text { broke }=\text { FALSE }
\end{aligned}
$$

It is not required that all state variables are given an initial value.

\subsection{Invariants}

It is important that the Black Jack dealer never allows broke and hitme to be true simultaneously (similarly stand and hitme must not be true simultaneously, neither must idle and hitme, etc.). These conditions are examples of invariants, and they specify a subset of the state space containing the "legal" states to enter during execution.

Invariants (and protocols introduced in Sect. 5.7) are stated explicitly by the designer to express properties of the design, for example:

\section{INVARIANT}

$\neg($ broke $\wedge$ hitme $) \wedge \neg($ idle $\wedge$ hitme $) \wedge \neg($ stand $\wedge$ hitme $) \wedge \neg($ broke $\wedge$ stand $)$

Invariants do not influence the behavior of a design, and removing them from a design description does not change the computation. This redundancy is quite similar to the use of declarations in high-level languages, for example Pascal: it is 
used to check consistency between the declaration and use of various quantities. There is, however, an important difference. Normally, declarations are checked syntactically by compilers; this is not possible for invariants and protocols which express dynamic properties. Instead, one could check these

- by an informal, but convincing argument,

- by a simulation, where the design is executed on specific input data,

- by enumerating and checking all possible states and transitions,

- by a formal proof.

The emphasis in this tutorial is on verification based on formal proofs supported by a mechanical theorem prover. One can verify that a given predicate $I$ is an invariant, by showing that:

1. $I$ holds in the initial state, and

2. for each transition $T$, if $I$ holds before executing $T$, then $I$ also holds afterwards.

This approach was originally proposed by Floyd for software verification [5]. The second condition is established by assuming that the invariant holds for the values of the state variables prior to executing the transition (called the prestate) and proving that the invariant holds for the values of the state variables afterwards (called the post-state). Consider, for example, the transition

$$
\ll \neg \text { idle } \wedge \neg \text { cardready } \wedge \text { hitme } \rightarrow \text { hitme: }=\text { FALSE } \gg
$$

This transition certainly maintains the invariant: $\neg($ idle $\wedge$ hitme $)$, because the transition only changes hitme to false; this can never violate the invariant.

The approach sketched above suggests that the verification is done by considering the transitions one at a time, and showing the implications separately for each transition. Note that the verification of a design is thereby reduced to a number of independent steps, one for each transition. This is important for mechanized verification, because a large design is handled by a number of (relatively) simple proofs instead of a few large and complicated ones. This is essentially what is done by the mechanical tools ST2LP and LP. For each transition, T, the translator generates a verification condition of the form:

$$
C: \quad I(\text { pre }) \wedge T(\text { pre }, \text { post }) \Rightarrow I(\text { post })
$$

where $T$ (pre, post) is a predicate corresponding to a transition $T$. LP then tries to verify each of the verification conditions.

\subsection{Integers and Subranges}

In addition to the boolean state variables used above, a design may contain state variables of type integer or a subrange of integer values, for example:

TYPE cardval $=[1 . .10]$

This specifies a type named cardval consisting of the integers in the range 1 to 10. Aces are represented by the value 1 : 
STATIC ace $=1$

A type is used to specify the value range of a state variable, for example:

STATE card: cardval

The state variable card may hold any of the values indicated by the type cardval, in this case the range 1 to 10 . This is a transition using such a state variable:

$\ll$ card $\neq$ ace $\rightarrow$ count $:=$ count + card $\gg$

It is important to check that the value assigned to a state variable is in the domain indicated by the type of the state variable. For example, the state variable card must only be assigned values in the range 1 to 10 . In many programming languages this check is done at run-time by executing a check every time a new value is assigned. However, for a design language like SYNCHRONIZED TRANSITIONS where many designs are realized as circuits, it is often worthwhile to do the checking before executing the design. When this is possible, there is no need to do the checking during execution. The specification of a range is rather similar to an invariant. The range is also redundant information and it cannot be checked statically. In fact, the range information can be transformed into an invariant and verified together with other invariants. The tools supporting verification of designs in SYNCHRONIZED TRANSITIONS are capable of automatically generating such range invariants and other similar conditions ensuring against indexing arrays out of bounds, division by zero, etc. These invariants are called well-formedness invariants. Well-formedness invariants for the Black Jack design are discussed in Sect. 5.11.

\subsection{Records}

Boolean, integer, and subrange are simple types. Structured types like records and arrays are used to group associated values, e.g., a record typically describes a number of different attributes of a data item. In the Black Jack example, the dealer's hand is described as a record with two components, a flag that indicates that the hand has at least one ace, and the accumulated score (where aces are counted as 1's):

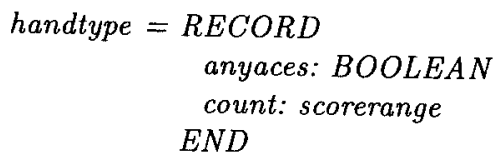

If anyaces is true the dealer might count one of the aces as 11 , thereby obtaining a score of count +10 (he will never count 2 aces as 11).

As it is the case in Pascal and many other high-level programming languages the individual components of a variable, $c$, of type record are obtained by giving the name of the state variable concatenated with the name of the component,
e.g., c.anyaces, as in the transition $\ll$ card $=$ ace $\rightarrow$ c.anyaces $:=$ TRUE $\gg$ 


\subsection{Functions}

Functions describe mappings from a list of parameters to a value, for example:

FUNCTION evallow(h: handtype): scorerange

RETURN h.count

FUNCTION evalhigh(h: handtype): extscorerange

RETURN h.count +IF h.anyaces THEN 10 ELSE 0

Functions can be used in all kinds of expressions both in transitions and to describe invariants:

INVARIANT broke $\Rightarrow$ evallow(hand) $>21$

\subsection{Protocols}

Protocols are predicates on pairs of states, pre, post, defining a restriction on the allowable transitions between states (to ones where the pre- and post-state satisfy the predicate). The following is an example of a protocol, stating that $x$ does not change.

$$
\text { PROTOCOL x.pre }=x . \text { post }
$$

x.pre denotes the value of $x$ in the pre-state and similarly x.post is the value of $x$ in the post-state. The four-phase handshake protocol is specified as follows.

$$
\begin{aligned}
& \text { FUNCTION fourphase(a, b: BOOLEAN): BOOLEAN }
\end{aligned}
$$

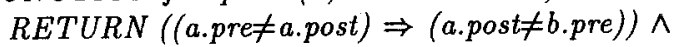

$$
\begin{aligned}
& ((\text { b.pre } \neq \text { b.post }) \Rightarrow(\text { b.post }=\text { a.pre }))
\end{aligned}
$$

To meet the protocol fourphase, a must get the value of $\neg b$ (or be unchanged), and when $b$ changes it must get the value of $a$. For instance, idle and cardready follows this protocol:

\section{PROTOCOL fourphase(idle, cardready)}

Like invariants, protocols are verified mechanically. The verification condition, $C$, given in Sect. 5.3, must be extended to also include verification of protocols. Assume that a design has the protocol $P$ and invariant $I$, then for each transition, $T$, the translator ST2LP generates a verification condition of the form:

$$
I(\text { pre }) \wedge T(\text { pre }, \text { post }) \Rightarrow I(\text { post }) \wedge P(\text { pre }, \text { post })
$$

\subsection{The Asynchronous Combinator}

The asynchronous combinator is used to describe the composition of a number of independent transitions. Consider for example:

- a transition for detecting that the dealer is broke

$$
\ll \neg \text { idle } \wedge \text { cardready } \wedge \text { evallow (hand) }>21 \rightarrow \text { broke: }=T R U E \gg
$$

- a transition for detecting that the dealer must stand 


$$
\begin{aligned}
& \ll \rightarrow i d l e \wedge \text { cardready } \wedge \text { (standrange }(\text { evallow }(\text { hand })) \vee \\
& \text { standrange }(\text { evalhigh }(\text { hand }))) \rightarrow \text { stand }:=T R U E \gg
\end{aligned}
$$

- a transition for determining that a new card can be dealt

$$
\begin{aligned}
& \ll \rightarrow \text { idle } \wedge \text { cardready } \wedge \text { evallow }(\text { hand }) \leq 16 \wedge \\
& \rightarrow \text { standrange }(\text { evalhigh }(\text { hand })) \rightarrow \text { hitme: }=\text { TRUE } \gg
\end{aligned}
$$

Each of these transitions (plus another handful) are needed to complete the design. They are combined using the asynchronous combinator $\|$.

\subsection{The Product Combinator}

The product combinator is used to factor a transition into a number of simpler transitions. Let $t_{1}, t_{2}$ be the following two transitions (where $v_{1}$ and $v_{2}$ are different state variables):

$$
\text { TRANSITION } t_{1} \ll c_{1} \rightarrow v_{1}:=e_{1} \gg \quad \text { TRANSITION } t_{2} \ll c_{2} \rightarrow v_{2}:=e_{2} \gg
$$

The product, $t_{1} * t_{2}$, is equivalent to the following transition:

$$
\ll c_{1} \wedge c_{2} \rightarrow v_{1}, v_{2}:=e_{1}, e_{2} \gg
$$

It is emphasized that the product composition of two transitions is not equivalent to the separate use of the transitions; the two transitions in a product operate simultaneously, as if it was a single transition (formed as shown above).

The product combinator makes it possible to separate transitions dealing with different aspects. The product combinator does not add expressive power, but in many cases it yields simpler and more comprehensible designs.

As an important special case, consider a transition description consisting of a precondition only, for example: $\ll \neg$ idle $\wedge$ cardready $\gg$. The product combinator can be used to factor this precondition out of a number of other transition descriptions, for example, the transitions given in Sect. 5.8:

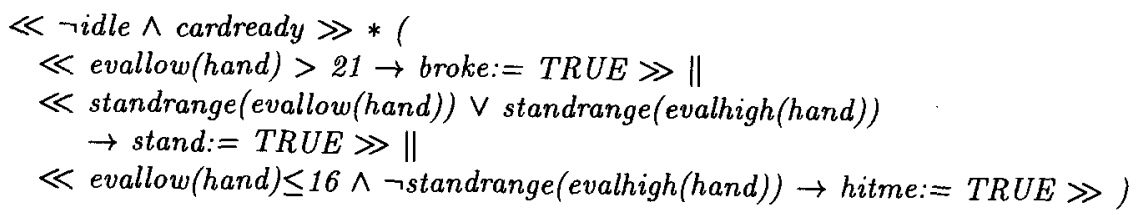

There is a third combinator, + , the synchronous combinator. It is used for describing a design where two or more enabled transitions are done simultaneously. UNITY has a similar combinator (written as $\|$ ). Section 6.3 describes an example using synchronous composition.

\subsection{Design of a Black Jack Dealer}

A hand is represented by the sum of the card values (where aces are counted as 1 's), and by a flag indicating that the hand has at least one ace. A low and high score of a hand are computed by the two functions evallow and evalhigh. The low score is computed by counting aces as 1's, i.e., it is just the accumulated score of the hand; the high score is computed by counting one ace (if any) as 11. If either the low or the high score is between 17 and 21 (determined by the function standrange) the dealer must stand. 
TYPE scorerange $=[0 . .26]$

$T Y P E$ extscorerange $=[0 . .36]$

FUNCTION evallow(c: handtype): scorerange RETURN c.count

FUNCTION evalhigh(c: handtype): extscorerange

RETURN c.count $+I F$ c.anyaces THEN 10 ELSE 0

FUNCTION standrange(s: extscorerange): BOOLEAN

$\operatorname{RETURN}(16<s) \wedge(s \leq 21)$

The following invariant captures the relationship between the state variables hand, hitme, stand, and broke:

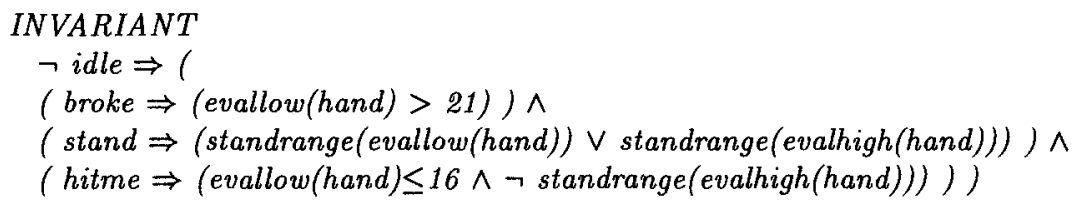

Sections 5.1-5.10 have explained most of the design description of the Black Jack dealer. In Fig. 1 all the pieces have been put together.

\subsection{Verifying the Black Jack Design}

The invariants $(I)$ and protocols $(P)$ specified in the design description can be verified using the translator (ST2LP) and the theorem prover (LP). The verification is divided into a number of separate proofs following the scheme introduced above, i.e., it must be verified that:

- $I$ holds in the initial state, and

- for each transition, $T: I($ pre $) \wedge T($ pre, post $) \Rightarrow I($ post $) \wedge P($ pre, post $)$

The translator generates the corresponding verification conditions: one for the initialization and one for each of the transitions in the design. All of these are verified independently, and it turns out that the theorem prover handles four of the conditions automatically, whereas (a little) manual assistance is needed to verify the remaining four conditions. It is too technical to show the proofs in more detail. In [16] it is shown how proofs for SYNChronized Transitions designs look like in LP.

To conclude that the design has the properties stated in the invariants and protocols, it must also be shown that the design description is well-formed. This is partly done by the translator which does the static checks, e.g., that the number of formal and actual parameters to functions match, that types are used consistently, etc. However, as mentioned previously, some checks cannot be done by ordinary static analysis, and in many programming languages such checks are instead done at run-time. The tools supporting verification of designs in SYNCHRONIZED TRANSITIONS automatically extract these dynamic properties and formulate them as (well-formedness) invariants $\left(I_{w f}\right)$, ready for verification 


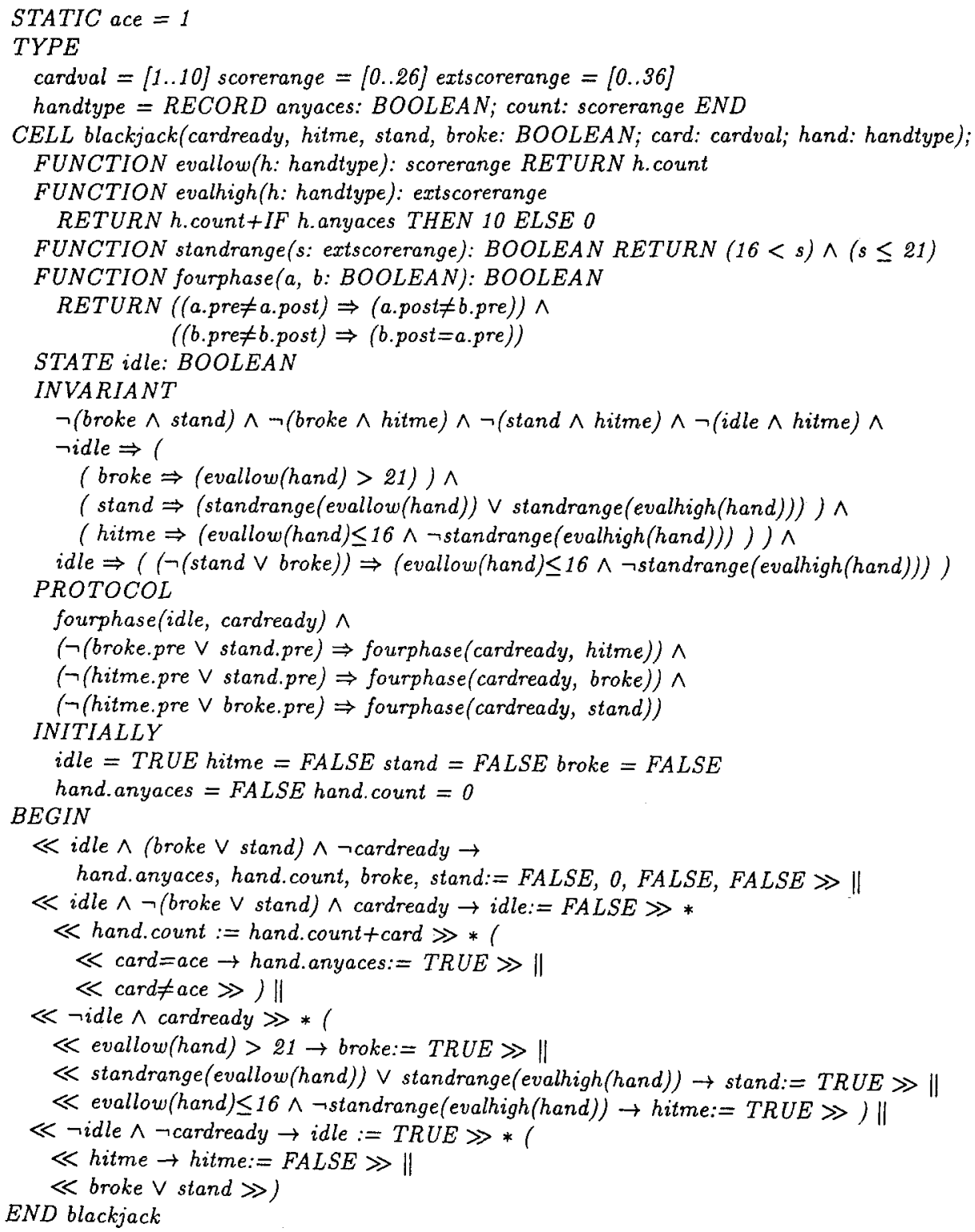

Fig. 1. Black Jack design in Synchronized Transitions. 
using the theorem prover. For instance, hand.count should keep a value in the integer range specified by its type, scorerange:

$$
I_{w f}: 0 \leq \text { hand.count } \leq 26
$$

The invariants for well-formedness initiate a set of proof obligations similar to those for the ordinary invariants and protocols, i.e., they must be verified in the initial state, and each transition should preserve them. Theoretically, they could be verified at the same time as the ordinary invariants by defining the invariant as the conjunction of the ordinary and well-formedness invariants, however, it is more practical to do these proofs independently. In this way it is possible to concentrate the initial efforts in the verification process on the ordinary invariants which often capture the more interesting and essential properties of the design.

For instance, the proof of the well-formedness invariant for the first transition needs manual assistance, whereas the proof of the ordinary invariant is handled automatically. If the invariants were verified at the same time, manual assistance is required, whereas, a separate verification makes it possible to verify the ordinary invariant automatically.

\subsection{Summary of Black Jack Design}

A number of invariants and protocols were verified for the Black Jack dealer:

- Relationships between the state variables broke, stand, hitme as explained in Sect. 5.3, e.g., that broke and hitme are not true simultaneously,

- relationships between the state variables broke, stand, hitme and the score of a hand as explained in Sect. 5.10, e.g., that the low score of a hand is greater than 21 when broke is true,

- that the Black Jack dealer obeys the four-phase handshake protocol as explained in Sect. 5.7,

- various well-formedness invariants as explained in Sect. 5.11, e.g., that state variables are not assigned a value outside the range specified by their type.

The verification of the properties listed above was performed with LP version $2.4 \mathrm{x}$ on a DEC Alpha $3000 / 400$ with $96 \mathrm{Mb}$ of memory. It takes 1:05 minutes for LP to read in the axiomatization of the Black Jack dealer. During this process the axioms are transformed into rewrite rules and facts are inter-normalized. The proofs of the ordinary (i.e., user supplied) invariants and protocols for the eight transitions take 3:28 minutes, whereas the proofs for the well-formedness invariants take 2:56 minutes. Four of the proofs of the ordinary invariants and protocols need manual assistance. Similarly, two of the proofs of the well-formedness invariants need manual assistance. None of the proofs need more than five user supplied LP commands. The two initialization proofs of the ordinary invariants and the well-formedness invariants take 0:26 minutes and 0:44 minutes, respectively, both proofs need manual assistance (less than ten LP commands).

Besides the CPU time used to do the proofs there is another significant factor: the "human" time used by the designer to supply manual assistance in 
the proofs; no attempt is made here to quantify this factor. Most of the manual assistance is needed to complete proof steps involving inequalities. This is quite typical, and proofs involving only equalities and booleans tend to require less manual assistance.

The design of the Black Jack dealer illustrates both the SYNCHRONIZED Transitions notation and the mechanical verification tools. For this example, the most interesting part of the verification is the range checking, i.e., that all assignments of new values to state variables are within the bounds given in the declaration of the state variables. This is a non-trivial and very important aspect of this design, and the tools are able to verify that no value will be out of bounds when this design is executed. Hence, there is no need to do a run-time check.

\section{Examples from the Benchmark Collection}

This section summarizes the verification of other examples from the TPCD benchmark collection.

\subsection{The Traffic Light Controller}

This is used as an explanatory example in [16]. It is shown that a design description in SYNCHRONIZED TRANSITIONS maintains the invariant:

$$
\text { EWlight }=\text { red } \vee \text { NSlight }=\text { red }
$$

Several versions of the design are also discussed in [14].

\subsection{The N-Bit Adder}

In [4] an efficient adder is described. The design (and efficiency) of this adder is based on a non-trivial invariant. In [13] it is shown how ST2LP and LP are used to verify a very efficient realization of an $N$-bit adder (without fixing the value of $N$ ).

\subsection{Min-Max}

This is mainly a descriptive benchmark. Below it is shown how to describe the design in SYNCHRONIZED TRANSITIONS. This example illustrates the use of synchronous composition of transitions (indicated by the operator + ). Synchronous composition is used to specify that two operations are always performed simultaneously (e.g., under control of a global clock); whereas, no such assumptions are made with asynchronous composition.

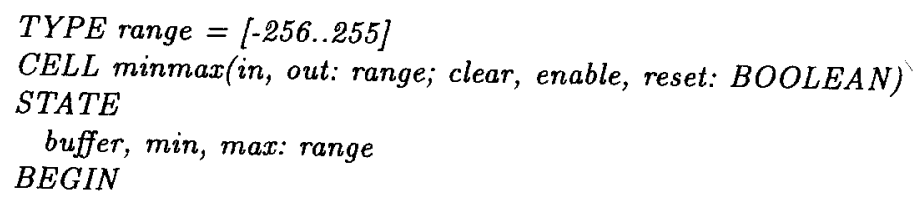




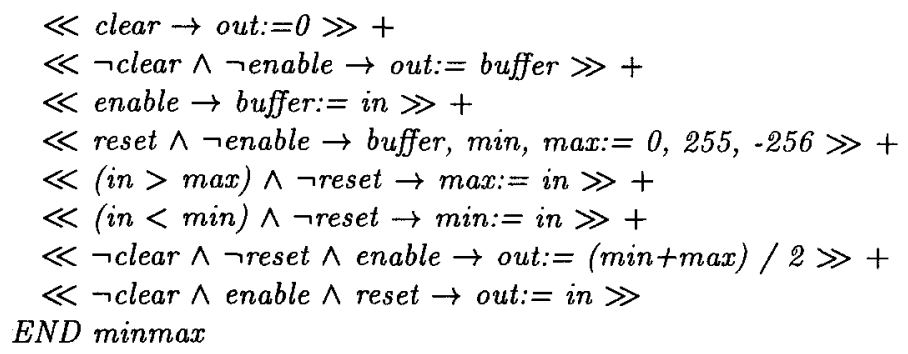

\subsection{The Arbiter}

This example is described in the paper [15] which also discusses the formal verification of mutual exclusion and a four-phase protocol. This arbiter design has a tree structure and this is reflected in the design description which is recursive. Such regular and other modular structures are verified using a localized verification technique where each cell is verified separately. By using this localized verification technique, it can be avoided that the verification of large designs blows up.

\subsection{The Tamarack Processor}

This is an example of implementation verification and hence very different from the other examples described in this tutorial. However, it is also possible to do mechanized implementation verification of designs described in SYNCHRONIZED TRANSiTions. There is a separate translator [12] that generates verification conditions for LP from two design descriptions: an abstract design (the specification) and a concrete design (the realization). This has been used to verify the Tamarack processor.

\subsection{The Stop-Watch, GCD, and FIFO}

These examples have all been described in Synchronized Transitions and various aspects have been verified using ST2LP and LP, see [14].

\section{$7 \quad$ Larger Designs}

It is rarely feasible to view a non-trivial design as one huge collection of transitions. In SYNCHRONIZED TrANSITIONS a design may be modularized by breaking it into cells. For example, the Black Jack dealer is a cell. The Black Jack dealer takes care of the game rules, etc., and it relies on a deck of cards that supplies (legal) cards at the right time. The deck of cards is another cell that works independently of the Black Jack cell. The dealer and the deck of cards communicates through shared variables, e.g., the dealer signals that it wants a new card using the state variable hitme, and the deck of cards signals that a 


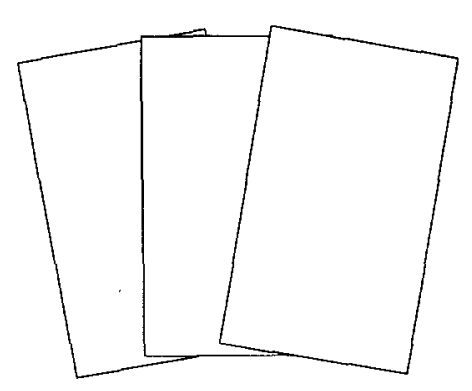

Black Jack Dealer

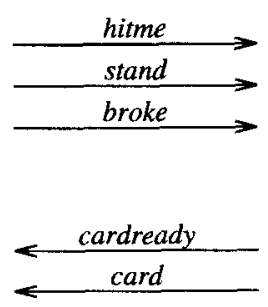

Deck of Cards

Fig. 2. Shared variable interface.

new card is ready using the state variable cardready. The interface consisting of shared variables is illustrated in Fig. 2. Breaking down a large design into cells is not only an aid when describing the design; it also makes the design easier to verify! This section presents a localized technique for verifying modular designs. The technique was originally proposed in [15] and it has been further developed in [17]. Each cell of the design is verified in isolation, showing what is called local correctness. Furthermore, it is verified that each cell does not violate the protocols and invariants of other cells. This is called non-interference, and it is verified solely on the basis of the interface of the cells, without considering their internal details. The localized verification technique is illustrated on the Black Jack dealer extended with a deck of cards cell.

The deck of cards cell must supply cards as the dealer is requesting them. This is the case if the dealer needs more cards for the hand (signalled with the hitme state variable), or when a game has finished and a new game must be started (signalled with the state variables stand and broke). The deck of cards cell uses the state variable cardready to tell when a new card is ready. The deck of cards cell may potentially break the four-phase protocol stated in the Black Jack dealer cell because it writes the state variable cardready. It is now shown how localized verification is used to verify that this is not the case.

\subsection{Local Correctness}

Local correctness is one part of the localized verification technique, where a cell is verified independently of its context. To do this, it is shown that all transitions of a cell maintain the invariant and protocol of the cell. For the Black Jack dealer cell this corresponds to the verification condition explained in Sect. 5. However, adding additional cells such as the deck of cards cell may introduce additional invariants and protocols describing properties of the interface. These additional properties are verified locally as described in Sect. 5. For instance, the deck of cards cell complies with the protocol: 


\author{
INTERNAL PROTOCOL \\ cardready.pre $\neq$ cardready.post $\Rightarrow$ \\ cardready.post $=\neg($ hitme.pre $\vee$ broke.pre $\vee$ stand.pre $)$
}

This protocol is called an internal protocol because it only holds for the transitions in the deck of cards cell; it need not (and does not) hold when transitions are executed in the Black Jack dealer cell.

The state variables in the two cells are shared; this allows the cells to communicate. But it also means that the transitions of one cell can violate the invariant and protocol of the other cell (interference). For example, the four-phase protocol between hitme and cardready is verified in the local correctness proof in the Black Jack cell (where hitme is changed), but the deck of cards cell writes cardready, and this might violate the four-phase protocol. Hence, local correctness is not sufficient to guarantee global correctness. In addition, it must be shown that cells do not interfere.

\title{
7.2 Non-interference
}

Non-interference is the complementary part of the localized verification technique. In this part, it is verified that invariants and protocols involving shared variables (parameters) are preserved. To verify that all invariants and protocols are maintained, it must be shown that the transitions in the deck of cards cell do not interfere with the invariants and protocols in the Black Jack cell, and it must also be shown that the transitions in the Black Jack cell do not interfere with the invariants and protocols in the deck of cards cell.

Instead of showing non-interference for each transition separately, it is done once for each cell. The key idea is to assume that the internal protocol, the invariant and the protocol of, e.g., the deck of cards cell capture the essential properties of all transitions in a single assertion. This is expressed in the two non-interference conditions presented below.

$$
\begin{aligned}
& I_{\text {deck }}(\text { post }) \wedge P_{\text {deck }}(\text { pre }, \text { post }) \wedge I P_{\text {deck }}(\text { pre }, \text { post }) \Rightarrow I_{b j}(\text { post }) \wedge P_{b j}(\text { pre }, \text { post }) \\
& I_{b j}(\text { post }) \wedge P_{b j}(\text { pre }, \text { post }) \wedge I P_{b j}(\text { pre }, \text { post }) \Rightarrow I_{\text {deck }}(\text { post }) \wedge P_{\text {deck }}(\text { pre }, \text { post })
\end{aligned}
$$

$(I, P$, and $I P$ are the invariant, protocol and internal protocol in the Black Jack cell (subscript $b j$ ) and deck of cards cell (subscript deck)). Note that the noninterference conditions avoid considering the individual transitions of the Black Jack dealer and deck of card cells. For example, it is shown from the internal protocol of the deck of cards cell that this cell does not interfere with the fourphase protocol $\left(P_{b j}\right)$. This is shown without considering the transitions of the deck of cards cell; as long as they comply with the internal protocol of the cell (this is verified as local correctness), the deck of cards cell will not violate the four-phase protocol.

In general, the cells of a design are arranged in a tree of cell instantiations. It is not necessary to verify the non-interference conditions between any two cells in the design, but only between "neighbor" cells in the instantiation tree. By showing the two non-interference conditions for all the neighbor pairs it is also 
(implicitly) shown that changes made in accordance with the protocol for one neighboring cell are also in accordance with the protocols for other neighbors. Therefore it circumvents the need to consider separately interferences that might result when parameters are passed through several levels. In [13] the technique is explained in more detail, and it is shown that the technique is sound.

\section{Experience}

We have used LP and Synchronized Transitions together to verify safety properties of several small, but interesting designs such as an arbiter[16], Chandy and Misra's efficient adder[4], a priority queue[17], and a set of building blocks for asynchronous circuits. We have also used these tools when teaching courses. Students have verified a series of examples such as the Black Jack design.

Designs described with SYNCHRONIZED TRANSITIONS can be manipulated and analyzed in a number of ways:

1. Design verification: Proving that transitions maintain properties formulated as invariant assertions.

2. Implementation verification: Proving that a concrete program, the realization, is a correct implementation of another program, the abstraction.

3. Simulation: Informally verifying a design by executing it with various input data.

4. Synthesis: Transforming a design into a circuit description, e.g., a netlist or a layout.

5. Verification of implementation conditions: Checking restrictions required for the realization to operate correctly.

Separate experimental tools support (1)-(5). This paper has concentrated on (1), for which LP is used together with a translator that translates a design description into a set of verification conditions. A similar translator for (2) is under development[12].

Our initial experience with LP and SYNCHRONIZED TRANSITIONS is encouraging. We believe that it is currently realistic to design moderate-sized circuits in a high-level language supported by both verification and synthesis tools.

Current techniques and tools for formal verification are not sufficiently refined for widespread application, but the potential benefits are so significant that we think they must be seriously investigated. When formal verification works it corresponds to an exhaustive simulation where a certain property is ensured for all combinations of inputs in all states. Since exhaustive simulation is rarely feasible in practice, formal verification has the potential of improving the quality of integrated circuit design.

\subsection{Availability of Tools}

LP is written in CLU and runs under Unix. Release 2.4 is currently available free of charge, and without a license, by anonymous ftp from larch.lcs.mit.edu. 
Prospective users can retrieve an executable version of LP, along with a supporting run-time library containing sample axiom sets and proofs, for DEC-stations, Sparc-stations, MIPS machines, Vaxes, or Sun-3 workstations, and soon DEC Alpha. Source code is also available.

The tools supporting SYNCHRONIZED TRANSITIONS are still undergoing development. They are written in $\mathrm{C}$ and run under Unix and can be obtained by anonymous ftp from ftp.id.dth.dk or by contacting the authors.

\section{Acknowledgements}

The work reported in this paper has been supported by The Danish Technical Research Council and by Digital Equipment Corporation through an external research grant. The authors are grateful to Stephen Garland and John Guttag from MIT, Cambridge, USA who have contributed significantly to the approach described in this paper.

\section{References}

1. Robert S. Boyer and J. Strother Moore. A Computational Logic. Academic Press, 1979.

2. Robert S. Boyer and J. Strother Moore. A Computational Logic Handbook. Academic Press, 1988.

3. Randal E. Bryant. Can a simulator verify a circuit? In Formal Aspects of VLSI Design, pages 125-136. North-Holland, 1985.

4. K. Mani Chandy and Jajadev Misra. Parallel Program Design: A Foundation. Addison-Wesley, 1988.

5. R.W. Floyd. Assigning meanings to programs. In J.T. Schwartz, editor, Proceedings of the Symposium in Applied Mathematics, volume 19, pages 19-32. American Mathematical Society, 1967.

6. Michael P. Fourman and Robert L. Harris. Lambda - logic and mathematics behind design automation. In Proceedings of the 26th ACM/IEEE design Automation Conference. ACM, 1989.

7. Stephen J. Garland and John V. Guttag. An overview of LP: the Larch Prover. In Proceedings of the Third International Conference on Rewriting Techniques and Applications. Springer-Verlag, 1989.

8. Mike Gordon. Why higher-order logic is a good formalism for specifying and verifying hardware. In Formal Aspects of VLSI Design, pages 153-177. NorthHolland, 1985.

9. Aarti Gupta. Formal hardware verification methods: A survey. Formal Methods in Systems Design, 1(2/3):151-238, October 1992.

10. John V. Guttag, James J. Horning with S.J. Garland, K.D. Jones, A. Modet, and J.M. Wing. Larch: Languages and Tools for Formal Specification. Springer-Verlag Texts and Monographs in Computer Science, 1993. ISBN 0-387-94006-5, ISBN 3-540-94006-5.

11. Warren A. Hunt. FM8501: A verified microprocessor. In From HDL Descriptions to Guaranteed Correct Circuit Designs, pages 85-114. North-Holland, 1986.

12. Niels Maretti. Mechanized verification of refinement. In Proceedings from TPCD '94, 1994. To appear. 
13. Niels Mellergaard. Mechanized Design Verification. PhD thesis, Department of Computer Science, Technical University of Denmark, 1994.

14. Jørgen Staunstrup. A Formal Approach to Hardware Design. Kluwer Academic Publishers, 1994.

15. Jørgen Staunstrup, Stephen J. Garland, and John V. Guttag. Localized verification of circuit descriptions. In Proceedings of the Workshop on Automatic Verifcation Methods for Finite State Systems, LNCS 407. Springer Verlag, 1989.

16. Jørgen Staunstrup, Stephen J. Garland, and John V. Guttag. Mechanized verification of circuit descriptions using the Larch Prover. In V. Stavridou and T. Melham, editors, Proceedings of the IFIP WG 10.2 International Conference on Theorem Provers in Circuit Design: Theory, Practice and Experience, pages 277300. Elsevier, 1992.

17. Jørgen Staunstrup and Niels Mellergaard. Localized verification of modular designs. Formal Methods in System Design, 1994. accepted for publication. 